\title{
Poisson-de Rham homology of hypertoric varieties and nilpotent cones
}

\author{
Nicholas Proudfoot 1 \\ Department of Mathematics, University of Oregon, Eugene, OR 97403 \\ Travis Schedler \\ Department of Mathematics, University of Texas, Austin, TX 78712
}

\begin{abstract}
We prove a conjecture of Etingof and the second author for hypertoric varieties, that the Poisson-de Rham homology of a unimodular hypertoric cone is isomorphic to the de Rham cohomology of its hypertoric resolution. More generally, we prove that this conjecture holds for an arbitrary conical variety admitting a symplectic resolution if and only if it holds in degree zero for all normal slices to symplectic leaves.

The Poisson-de Rham homology of a Poisson cone inherits a second grading. In the hypertoric case, we compute the resulting 2-variable Poisson-de Rham-Poincaré polynomial, and prove that it is equal to a specialization of an enrichment of the Tutte polynomial of a matroid that was introduced by Denham [Den01]. We also compute this polynomial for S3-varieties of type A in terms of Kostka polynomials, modulo a previous conjecture of the first author, and we give a conjectural answer for nilpotent cones in arbitrary type, which we prove in rank less than or equal to 2 .
\end{abstract}

\section{Introduction}

Let $X$ be a Poisson variety over C. Etingof and the second author [ES10a] define a right D-module $M(X)$, and define the Poisson-de Rham homology group $\operatorname{HP}_{k}(X)$ to be the cohomology in degree $-k$ of the derived pushforward of $M(X)$ to a point. If $X$ is affine, then $\mathrm{HP}_{0}(X)$ coincides with the zeroth Poisson homology of $\mathbf{C}[X]$, but $\mathrm{HP}_{*}(X)$ does not directly relate to higher Poisson homology. If $X$ is smooth and symplectic, then $M(X)$ is naturally isomorphic to the right $\mathrm{D}$-module $\Omega_{X}$ of volume forms on $X$, and therefore we have an isomorphism $\mathrm{HP}_{k}(X) \cong \mathrm{H}^{\operatorname{dim} X-k}(X ; \mathbf{C})$. The next natural case to consider is when $X$ is singular but admits a conical symplectic resolution $2 \rho: \tilde{X} \rightarrow X$; examples include hypertoric varieties, symmetric schemes of Kleinian singularities (more generally, Nakajima quiver varieties), nilpotent cones (more generally, S3-varieties), and certain slices to Schubert varieties in the affine Grassmannian [BPW, §2]. In this case, Etingof and the second author [ES13, 1.3.1] conjecture that $M(X)$ is (noncanonically) isomorphic to $\rho_{*} \Omega_{\tilde{X}}$, and therefore that $\operatorname{HP}_{k}(X) \cong \mathrm{H}^{\operatorname{dim} X-k}(\tilde{X} ; \mathbf{C})$.

\footnotetext{
${ }^{1}$ Supported by NSF grant DMS-0950383.

${ }^{2} \mathrm{~A}$ precise definition of a conical symplectic resolution is given at the beginning of Section 3 .
} 
In this paper, we prove this conjecture for hypertoric varieties. More generally, we show that if the vector space isomorphism holds when $k=0$ not just for $X$, but also for all normal slices to symplectic leaves of $X$, then the D-module isomorphism $M(X) \cong \rho_{*} \Omega_{\tilde{X}}$ holds, as well (Theorem 4.1). These vector space isomorphisms have already been established for hypertoric varieties by the first author [Pro14, 3.2], therefore Theorem 4.1 applies.

Part of the structure of a conical symplectic resolution is an action of $\mathbf{C}^{\times}$on $X$ with respect to which the Poisson bracket is homogeneous. The right $\mathrm{D}$-module $M(X)$ is weakly $\mathbf{C}^{\times}$-equivariant, and this induces a second grading on $\operatorname{HP}_{*}(X)$, which we call the weight grading. We prove a general result (Theorem 5.1 and its corollaries) that computes $M(X)$, with its weight grading, in terms of the degree zero Poisson homology of the slices. Let $P_{X}(x, y)$ be the Poincaré polynomial of $\operatorname{HP}_{*}(X)$, where $x$ encodes homological degree and $y$ encodes weight. When $X$ is a hypertoric variety, we show that $P_{X}(x, y)$ is equal to a specialization of a polynomial studied by Denham [Den01] that encodes the dimensions of the eigenspaces of the combinatorial Laplacian of a matroid (Theorem 6.1), which is closely related to the Tutte polynomial of the associated hyperplane arrangement. When $X$ is an S3-variety of type A, we similarly compute $P_{X}(x, y)$ in terms of Kostka polynomials (Proposition 17.1), modulo a conjecture that appears in [Pro14, 3.4]. Finally, we give a conjectural description of $P_{X}(x, y)$ where $X$ is the nilpotent cone in arbitrary type (Conjecture 8.4), and prove it in certain cases.

Acknowledgments: The first author would like to thank G. Denham, C. Mautner, and V. Ostrik for their help with this project, and in particular we thank Mautner for help with Lemmas 3.3 and 3.4. The second author would like to thank P. Etingof for useful discussions, particularly about Proposition 2.1 and Conjecture 8.4, and for helpful comments on an earlier version. We are grateful to G. Lusztig for suggesting the formula of Conjecture 8.4, and for introducing us to the material in Remark 8.10 .

\section{The twistor family}

Let $X$ be a normal, irreducible, affine Poisson variety of finite type over C. Let $\rho: \tilde{X} \rightarrow X$ be a projective symplectic resolution, equipped with a particular choice of ample line bundle on $\tilde{X}$. Kaledin extends $\rho$ to a projective map $\rho: \tilde{\mathfrak{X}} \rightarrow \mathfrak{X}$ of schemes over the formal disk $\Delta:=\operatorname{Spec} \mathbf{C}[[t]]$, where over the closed point $0 \in \Delta$ we have

$$
\tilde{\mathfrak{X}}_{0} \cong \tilde{X} \quad \text { and } \quad \mathfrak{X} \cong X
$$

Furthermore, he shows that $\mathfrak{X}$ is normal and flat over $\Delta$, and that over the generic point, $\rho$ restricts to an isomorphism of smooth, affine, symplectic varieties [Kal08, 2.2 and 2.5]. This family of maps over $\Delta$ is called the twistor family.

Let $M:=M(X)$, and let $T:=\rho_{*} \Omega_{\tilde{X}}$ be the derived pushforward to $X$ of the right D- 
module of volume forms on $\tilde{X}$. By [Kal06, 2.11], $\rho$ is semismall, hence $T$ is also a right D-module (that is, the homology of $T$ is concentrated in degree zero). These extend naturally to right D-modules $\mathfrak{M}:=M(\mathfrak{X})$ and $\mathfrak{T}:=\rho_{*} \Omega_{\mathfrak{X}}$ on $\mathfrak{X}$. Let $\mathfrak{M}_{0}$ be the right D-module on $\mathfrak{X}$ obtained by killing $\mathbf{C}[[t]]$-torsion in $\mathfrak{M}$, and let $M_{0}$ be the restriction of $\mathfrak{M}_{0}$ to $X$. (In Theorem 4.1, we will show that, under suitable hypotheses, $M_{0}$ is isomorphic to $M$. However, we $a$ priori know only that $M_{0}$ is a quotient of $M$.)

We would like to perform the same construction on $\mathfrak{T}$ and $T$, but it is unnecessary: if we forget the Poisson structure, the family $\tilde{\mathfrak{X}}$ over $\Delta$ is locally trivial (that is, $\tilde{\mathfrak{X}}$ admits an open cover by trivial families over $\Delta$ ) [Nam08, 17], thus $\mathfrak{T}$ has no $\mathbf{C}[[t]]$-torsion. Since $M(X)$ equals the canonical D-module of volume forms when $X$ is smooth and symplectic [ES10a, 2.6], the right D-modules $\mathfrak{M}_{0}, \mathfrak{M}$, and $\mathfrak{T}$ are all isomorphic at the generic fiber.

Proposition 2.1 The semisimplification of $M_{0}$ is (noncanonically) isomorphic to $T$.

Proof: Since $\mathfrak{M}_{0}$ and $\mathfrak{T}$ are isomorphic at the generic fiber, the semisimplifications of $M_{0}$ and $T$ must be isomorphic (as they have the same class in the Grothendieck group of holonomic D-modules on $X$ ). But $T$ is semisimple by the decomposition theorem [BBD82, 6.2.5], so it must be isomorphic to the semisimplification of $M_{0}$.

\section{$3 \quad$ Rigidity}

We now add the hypothesis that $\rho: \tilde{X} \rightarrow X$ is conical, which means the following:

- $X$ and $\tilde{X}$ are both equipped with actions of the multiplicative group $\mathbf{C}^{\times}$, and the map $\rho$ is equivariant.

- The action of $\mathbf{C}^{\times}$induces a non-negative grading on $\mathbb{C}[X]$, with only the constant functions in degree zero.

- The Poisson bracket on $X$ (equivalently the symplectic form on $\tilde{X}$ ) is homogeneous for the action of $\mathbf{C}^{\times}$.

Our aim in this section is to prove that $\operatorname{Ext}^{1}(T, T)=0$, and therefore that $M_{0}$ is in fact isomorphic to $T$. We accomplish this in two steps, first showing that all summands supported on a single leaf have no self-extensions, and then showing that there can be no extensions between summands of $T$ supported on different leaves.

For the first step, we prove more generally that all topological local systems on a leaf are semisimple. We use the term local system to mean an $\mathcal{O}$-coherent right $\mathcal{D}$-module (equivalently, a vector bundle with a flat connection) on a locally closed smooth subvariety. We use the term topological local system to mean a representation of the fundamental 
group of such a subvariety. By the Riemann-Hilbert correspondence, the latter are equivalent to the former when we require that the connection has regular singularities. All of the local systems we consider will have regular singularities.

Proposition 3.1 All finite-rank topological local systems on a leaf $S \subset X$ are semisimple.

Remark 3.2 Proposition 3.1 does not require that $X$ admit a symplectic resolution, but only that it be conical and be a symplectic variety in the sense of Beauville [Bea00, which means that the Poisson bracket on the regular locus of $X$ is nondegenerate and the inverse meromorphic symplectic form extends to a (possibly degenerate) 2-form on some (equivalently every) resolution of $X$. Such varieties include, for example, quotients of symplectic varieties by finite groups acting symplectically [Bea00, 2.4], which often do not admit symplectic resolutions (see, e.g., [BS13]).

Proof of Proposition [3.1; Let $Y$ be the normalization of the closure of $S$ in $X$. Then $Y$ is a symplectic variety in the sense of Beauville [Kal06, 2.5], and the conical action on $X$ induces a conical action on $Y$. The regular locus $Y_{\text {reg }}$ is birational to $S$ and isomorphic away from a subvariety of codimension 2; in particular, the fundamental groups of $S$ and $Y_{\text {reg }}$ are isomorphic. Thus it is sufficient to prove that every finite-rank topological local system on $Y_{\text {reg }}$ is trivial.

Since $Y_{\text {reg }}$ is a quasiprojective variety, $\pi_{1}\left(Y_{\text {reg }}\right)$ is finitely generated (this follows, for example, from the finite triangulability of [Łoj64]). In the situation at hand, Namikawa has proved that the profinite completion $\hat{\pi}_{1}\left(Y_{\text {reg }}\right)$ is finite [Nam. By a theorem of Grothendieck Gro70], the map $\pi_{1}\left(Y_{\text {reg }}\right) \rightarrow \hat{\pi}_{1}\left(Y_{\text {reg }}\right)$ induces an equivalence of categories of finite-dimensional representations, hence the category of finite-dimensional representations of $\pi_{1}\left(Y_{\mathrm{reg}}\right)$ (equivalently, the category of finite-rank topological local systems on $\left.Y_{\text {reg }}\right)$ is semisimple.

Let $S \subset X$ be a symplectic leaf, and let $i: \bar{S} \backslash S \rightarrow X$ be the inclusion of the boundary of $S$. Let $K_{S}:=H^{\operatorname{codim} S} \rho_{*} \Omega_{\rho^{-1}(S)}$, which is a local system on $S$ with regular singularities. Since the resolution $\rho$ is semismall [Kal06, 2.11], the decomposition theorem [BBD82, 6.2.5] yields

$$
T \cong \bigoplus_{S} \mathrm{IC}\left(S ; K_{S}\right)
$$

By Proposition 3.1, we conclude that $\operatorname{Ext}^{1}\left(K_{S}, K_{S}\right)=0$, and therefore that we have $\operatorname{Ext}^{1}\left(\operatorname{IC}\left(S ; K_{S}\right), \operatorname{IC}\left(S ; K_{S}\right)\right)$ 0 for all $S$.

It remains to show that there are no extensions between summands on different leaves. We do this using the following two lemmas 3

\footnotetext{
${ }^{3}$ The authors thank Carl Mautner for explaining the following two lemmas and their proofs.
} 
Lemma 3.3 The complex $i_{*} i^{*} \mathrm{IC}\left(S ; K_{S}\right)$ of right $D$-modules is concentrated in degrees $\leq-2$.

Proof: It is a standard property of intermediate extensions of local systems that $i^{*} \operatorname{IC}\left(S ; K_{S}\right)$ is concentrated in negative degrees. Since $i$ is a closed embedding, $i_{*}$ is exact, and thus $i_{*} i^{*} \mathrm{IC}\left(S ; K_{S}\right)$ is concentrated in negative degrees. Therefore we only have to show that $\mathrm{H}^{-1} i_{*} i^{*} \mathrm{IC}\left(S ; K_{S}\right)=0$.

For a contradiction, let $S^{\prime}$ be a maximal symplectic leaf in the closure of $S$ on which $\mathrm{H}^{-1} i_{*} i^{*} \mathrm{IC}\left(S ; K_{S}\right)$ is supported, and let $j_{S^{\prime}}: S^{\prime} \rightarrow X$ the inclusion. Then

$$
\mathrm{H}^{-1} j_{S^{\prime}}^{*} i_{*} i^{*} \mathrm{IC}\left(S ; K_{S}\right)=\mathrm{H}^{-1} j_{S^{\prime}}^{*} \mathrm{IC}\left(S ; K_{S}\right)
$$

is a local system on $S^{\prime}$. By our assumption, the stalk of $\operatorname{IC}\left(S ; K_{S}\right)$ at every point of $S^{\prime}$ has nonzero cohomology in degree $-\operatorname{dim} S^{\prime}-1$.

Choose a point $x \in S^{\prime}$. The stalk $\mathrm{IC}\left(S ; K_{S}\right)_{x}$ is a summand of $T_{x}$. But $\mathrm{H}^{*}\left(T_{x}\right)$ is the pushforward to a point of the restriction of $\Omega_{\tilde{X}}$ to the fiber $\rho^{-1}(x)$. This is the same for the formal neighborhood (or an analytic neighborhood) of $\rho^{-1}(x)$, thus we obtain the shifted topological cohomology $\mathrm{H}^{*+\operatorname{dim} X}\left(\rho^{-1}(x) ; \mathbf{C}\right)$ of the fiber. By $[\mathrm{Kal09}, 1.9], \mathrm{H}^{*}\left(\rho^{-1}(x)\right.$; $\left.\mathbf{C}\right)$ is concentrated in even degrees, and hence the same is true for $\mathrm{H}^{*}\left(T_{x}\right)$. Since $\operatorname{dim} S^{\prime}$ is even, this gives us a contradiction.

Lemma 3.4 Let $S \neq S^{\prime}$ be symplectic leaves of $X$. Then $\operatorname{Ext}^{1}\left(\operatorname{IC}\left(S ; K_{S}\right), \operatorname{IC}\left(S^{\prime}, K_{S^{\prime}}\right)\right)=0$.

Proof: Assume first that $S$ is not contained in the closure of $S^{\prime}$. Thus, $S$ is disjoint from the closure of $S^{\prime}$. Let $j_{S}: S \rightarrow X$ and $i_{S}: \bar{S} \backslash S \rightarrow X$ be the inclusions. Then $j_{S}^{*} \operatorname{IC}\left(S^{\prime}, K_{S^{\prime}}\right)=0$. We have the standard exact triangle

$$
\rightarrow\left(j_{S}\right) ! K_{S} \rightarrow \mathrm{IC}\left(S ; K_{S}\right) \rightarrow\left(i_{S}\right)_{*} i_{S}^{*} \mathrm{IC}\left(S ; K_{S}\right) \rightarrow
$$

Apply $\operatorname{Hom}\left(-, \mathrm{IC}\left(S^{\prime}, K_{S^{\prime}}\right)\right)$, and we obtain in the long exact sequence,

$$
\begin{aligned}
\rightarrow \operatorname{Ext}^{1}\left(\left(i_{S}\right)_{*} i_{S}^{*} \operatorname{IC}\left(S ; K_{S}\right), \operatorname{IC}\left(S^{\prime}, K_{S^{\prime}}\right)\right) \rightarrow \operatorname{Ext}^{1}(\mathrm{IC}(S ; & \left.\left.K_{S}\right), \operatorname{IC}\left(S^{\prime}, K_{S^{\prime}}\right)\right) \\
& \rightarrow \operatorname{Ext}^{1}\left(\left(j_{S}\right)_{!} K_{S}, \operatorname{IC}\left(S^{\prime}, K_{S^{\prime}}\right)\right) \rightarrow .
\end{aligned}
$$

We want to show that the middle term is zero. By adjunction, since $j_{S}^{*} \operatorname{IC}\left(S^{\prime}, K_{S^{\prime}}\right)=0$, the last term is zero. It suffices therefore to show that the first term is zero. However, by Lemma 3.3. $\left(i_{S}\right)_{*} i_{S}^{*} \mathrm{IC}\left(S ; K_{S}\right)$ has cohomology concentrated in degrees $\leq-2$, whereas $\operatorname{IC}\left(S^{\prime}, K_{S^{\prime}}\right)$ is a D-module (in degree zero). Therefore, the first term is also zero 4

\footnotetext{
${ }^{4}$ More generally, for any triangulated category with a $t$-structure, if $M$ is a complex whose cohomology is concentrated in negative degrees and $N$ is a complex whose cohomology is concentrated in nonnegative degrees, then $\operatorname{Hom}(M, N)=0$.
} 
Next assume $S$ is contained in the closure of $S^{\prime}$. Since $S \neq S^{\prime}, S^{\prime}$ is not contained in the closure of $S$. In this case, applying Verdier duality,

$$
\operatorname{Ext}^{1}\left(\mathrm{IC}\left(S ; K_{S}\right), \operatorname{IC}\left(S^{\prime}, K_{S^{\prime}}\right)\right)=\operatorname{Ext}^{1}\left(\mathbf{D} \operatorname{IC}\left(S^{\prime}, K_{S^{\prime}}\right), \mathbf{D I C}\left(S ; K_{S}\right)\right)
$$

But, since $\Omega_{\tilde{X}}$ is self-dual, so is $T$, and hence $\mathbf{D I C}\left(S ; K_{S}\right)=\operatorname{IC}\left(S ; \mathbf{D} K_{S}\right)$ is a summand of $T$. Therefore, $\operatorname{IC}\left(S ; \mathbf{D} K_{S}\right) \cong \operatorname{IC}\left(S ; K_{S}\right)$, and the same holds for $S^{\prime}$. Thus we again have $\operatorname{Ext}^{1}\left(\mathrm{IC}\left(S ; K_{S}\right), \operatorname{IC}\left(S^{\prime}, K_{S^{\prime}}\right)\right)=0$.

Putting together Lemma 3.4 and Proposition 3.1, (3.1) immediately implies:

Proposition 3.5 The D-module $T$ is rigid; that is, $\operatorname{Ext}^{1}(T, T)=0$.

The following corollary is an immediate consequence of Propositions 2.1 and 3.5 .

Corollary 3.6 $M_{0}$ is isomorphic to $T$.

\section{The main theorem}

For each leaf $S$, choose a point $s \in S$, and let $X_{S}$ be a formal slice to $S$ at $s$. Then the base change of $\rho$ along the inclusion of $X_{S}$ into $X$ induces a projective symplectic resolution $\tilde{X}_{S} \rightarrow X_{S}$. The following theorem asserts that, if [ES13, Conjecture 1.3.(a)] holds for each $X_{S}$, then [ES13, Conjecture 1.3.(c)] holds for $X$.

Theorem 4.1 Suppose that, for every leaf $S$ of $X$, $\operatorname{dim} \operatorname{HP}_{0}\left(X_{S}\right)=\operatorname{rk} K_{S}$. Then $M \cong T$.

Note that, in the above theorem, the isomorphism $M \cong T$ is not canonical. This can be corrected as follows. Let $i: S \rightarrow X$ be the inclusion, and let $L_{S}:=\mathrm{H}^{0}\left(i^{*} M\right)$ be the local system studied in [ES10a, §4.3] 5 The fiber $L_{S, s}$ of $L_{S}$ at the point $s$ is canonically isomorphic to the vector space $\mathrm{HP}_{0}\left(X_{S}\right)$ ES10a, 4.10].

Corollary 4.2 Under the hypothesis of Theorem 4.1, there is a canonical isomorphism $M \cong$ $\bigoplus_{S} \mathrm{IC}\left(S ; L_{S}\right)$, and a noncanonical isomorphism $L_{S} \cong K_{S}$ for each symplectic leaf $S$.

Proof: As explained in [ES10a, §4.3], the right D-modules $\mathrm{IC}\left(S ; L_{S}\right)$ are subquotients of $M$. Theorem 4.1 and Equation (3.1) together imply that $L_{S}$ is a subquotient of $K_{S}$ for all $S$. Since $\operatorname{rk} L_{S}=\operatorname{dim} \mathrm{HP}_{0}\left(X_{S}\right)=\operatorname{rk} K_{S}, K_{S}$ and $L_{S}$ are in fact isomorphic.

Consider the canonical adjunction morphism $M \rightarrow H^{0}\left(i_{*} L_{S}\right)$, which induces a surjection from $M$ to $\operatorname{IC}\left(S ; L_{S}\right)$. By Proposition 3.5, the map $M \rightarrow \bigoplus_{S} \mathrm{IC}\left(S ; L_{S}\right)$ is an isomorphism.

\footnotetext{
${ }^{5}$ It is not a priori clear that $L_{S}$ has regular singularities, though this will follow from Corollary 4.2
} 
Proof of Theorem 4.1; Let $N$ be the kernel of the surjection $M \rightarrow M_{0} \cong T$, so that we have a short exact sequence

$$
0 \rightarrow N \rightarrow M \rightarrow T \rightarrow 0
$$

of right D-modules on $X$. Assume for the sake of contradiction that the support of $N$ is nontrivial. It is necessarily a union of symplectic leaves; let $S$ be a maximal such leaf. Restrict to the formal neighborhood of the leaf $S$. Then $N, M$, and $T$ are local systems along $S$ (that is, upon restriction to a contractible analytic open neighborhood $U$ of every point of $S$, they become external tensor products of local systems on $U$ and D-modules on the normal slice). We can therefore make use of the exact restriction functor for such D-modules to the slice $X_{S}$ at $s$, given by $P \mapsto P_{S}:=i_{X_{S}}^{*} P[-\operatorname{dim} S]$ (for $i_{X_{S}}$ the inclusion of $X_{S}$ into the formal neighborhood of $S$ ), and we obtain the exact sequence

$$
0 \rightarrow N_{S} \rightarrow M_{S} \rightarrow T_{S} \rightarrow 0
$$

By functoriality and the definitions of $M$ and $T, M_{S}$ is isomorphic to $M\left(X_{S}\right)$, and $T_{S}$ is isomorphic to the derived pushforward of the canonical sheaf of $\tilde{X}_{S}$.

Let $\pi$ be the pushforward of $X_{S}$ to a point. We have

$$
\mathrm{H}^{-1} \pi_{*}\left(T_{S}\right) \cong \mathrm{H}^{\operatorname{dim} X_{S}-1}\left(\rho^{-1}(s) ; \mathbf{C}\right)=0
$$

by [Kal06, 2.12]. Also, $N_{S}$ is a delta-function D-module at $s$, so $\pi_{*} N_{S}$ is concentrated in degree zero. Thus, we obtain a short exact sequence

$$
0 \rightarrow \mathrm{H}^{0} \pi_{*}\left(N_{S}\right) \rightarrow \mathrm{H}^{0} \pi_{*}\left(M_{S}\right) \rightarrow \mathrm{H}^{0} \pi_{*}\left(T_{S}\right) \rightarrow 0
$$

We have

$$
\mathrm{H}^{0} \pi_{*}\left(T_{S}\right) \cong \mathrm{H}^{\operatorname{dim} X_{S}}\left(\rho^{-1}(s) ; \mathbf{C}\right)
$$

By assumption, we also have

$$
\operatorname{dim} \mathrm{H}^{0} \pi_{*}\left(M_{S}\right)=\operatorname{dim} \mathrm{HP}_{0}\left(X_{S}\right)=\operatorname{rk} K_{S}=\operatorname{dim} \mathrm{H}^{\operatorname{dim} X_{S}}\left(\rho^{-1}(s) ; \mathbf{C}\right)
$$

Thus $\mathrm{H}^{0} \pi_{*}\left(M_{S}\right)$ and $\mathrm{H}^{0} \pi_{*}\left(T_{S}\right)$ have the same dimension, and therefore $\mathrm{H}^{0} \pi_{*}\left(N_{S}\right)=0$. This means that $N_{S}=0$, which is a contradiction.

Let $\pi$ be the map from $X$ to a point. By definition, we have $\operatorname{HP}_{k}(X):=\mathrm{H}^{-k} \pi_{*}(M)$. By the de Rham theorem, we have an isomorphism $\mathrm{H}^{-k} \pi_{*}(T) \cong \mathrm{H}^{\operatorname{dim} X-k}(\tilde{X} ; \mathbf{C})$. Thus, as explained by Etingof and the second author [ES13, 1.3], Theorem 4.1 implies that the 
Poisson-de Rham homology of $X$ is isomorphic to the de Rham cohomology of $\tilde{X}$.

Corollary 4.3 Under the hypothesis of Theorem 4.1, we have a (noncanonical) isomorphism $\operatorname{HP}_{k}(X) \cong \mathrm{H}^{\operatorname{dim} X-k}(\tilde{X} ; \mathbf{C})$ for all $k$.

Corollary 4.4 Any two conical projective symplectic resolutions of $X$ have the same Betti numbers.

Remark 4.5 In fact, it is possible to show that any two conical projective symplectic resolutions of $X$ have canonically isomorphic cohomology rings; this follows from [Nam08, 25].

Example 4.6 Let $\mathcal{A}$ be a coloop-free, unimodular, rational, central hyperplane arrangement, and let $X(\mathcal{A})$ be the associated hypertoric variety [PW07, $\S 1$. Any simplification $\tilde{\mathcal{A}}$ of $\mathcal{A}$ determines a conical projective symplectic resolution $\tilde{X}(\tilde{\mathcal{A}})$ of $X(\mathcal{A})$. The symplectic leaves of $X(\mathcal{A})$ are indexed by coloop-free flats of $\mathcal{A}$, and the slice to the leaf indexed by $F$ is isomorphic to a formal neighborhood of the cone point of $X\left(\mathcal{A}_{F}\right)$, where $\mathcal{A}_{F}$ is the localization of $\mathcal{A}$ at $F[$ PW07, §2]. Hence the hypothesis of Theorem 4.1 is that, for every coloop-free flat $F$,

$$
\operatorname{dim} \operatorname{HP}_{0}\left(X\left(\mathcal{A}_{F}\right)\right)=\operatorname{dim} \mathrm{H}^{2 \operatorname{rk} F}\left(\tilde{X}\left(\tilde{\mathcal{A}}_{F}\right) ; \mathbf{C}\right)
$$

This is proved in [Pro14, 3.2], hence Theorem 4.1 holds for hypertoric varieties.

\section{$5 \quad$ Weights}

We assume throughout this section that the hypothesis of Theorem 4.1 is satisfied.

By homogeneity of the Poisson bracket, the vector space

$$
\operatorname{HP}_{0}(X) \cong \mathbf{C}[X] /\{\mathbf{C}[X], \mathbf{C}[X]\}
$$

inherits a grading from the action of $\mathbf{C}^{\times}$. Moreover, the D-module $M$ has a canonical weak $\mathbf{C}^{\times}$-equivariant structure, thus $\operatorname{HP}_{k}(X)=\mathrm{H}^{-k} \pi_{*}(M)$ is naturally graded for all $k$ (where $\pi$ is the map from $X$ to a point).

Let $n$ be the positive integer such that the Poisson bracket on $X$ has weight $-n$ (this weight must be negative since the bracket vanishes along $\rho^{-1}(0)$ in the resolution $\tilde{X}$ ). Suppose that, for every symplectic leaf $S$, the normal slice $X_{S}$ admits a conical $\mathbf{C}^{\times}$-action equipping the Poisson bracket on $X_{S}$ with the same weight $-n$. More generally, we can suppose $X_{S}$ to be equipped with a vector field $\xi$ such that $L_{\xi} \pi_{X_{S}}=-n \pi_{X_{S}}$, where $\pi_{X_{S}}$ is the Poisson bivector (that is, we only require an infinitesimal action of $\mathbf{C}^{\times}$). In fact, this is no additional assumption: such a $\xi$ always exists by virtue of the Darboux-Weinstein decomposition $\hat{X}_{s} \cong$ $\hat{S}_{s} \hat{\times} X_{S}$ [Kal06, 2.3]. If $p: \hat{X}_{s} \rightarrow X_{S}$ is the projection, we may take $\xi=p_{*}\left(\left.\operatorname{Eu}_{\hat{X}_{s}}\right|_{\{0\} \times X_{S}}\right)$, 
where $\mathrm{Eu}_{\hat{X}_{s}}$ is the vector field for the $\mathbf{C}^{\times}$-action. However, we impose no requirement that the vector field $\xi$ be obtained in this way.

Let $\pi$ be the Poisson bivector on $X$, and let $\theta$ be any vector field such that $L_{\theta} \pi=c \pi$ for some $c \in \mathbf{C}$. Then the bracket of $\theta$ with any Hamiltonian vector field is again Hamiltonian, thus left multiplication by $\theta$ is an endomorphism of the right D-module $M(X)$. It is the zero endomorphism if and only if $\theta$ is Hamiltonian (in which case $c=0$ ). Since $\operatorname{IC}\left(S ; L_{S}\right)$ is (canonically) a quotient of $M(X)$, this also induces an endomorphism of $\operatorname{IC}\left(S ; L_{S}\right)$, and hence of $L_{S}$ and its fiber $L_{S, s}$. In the case of the Euler vector field $\mathrm{Eu}_{X}$, which is induced by an honest action of $\mathbf{C}^{\times}$, this endomorphism must be semisimple. By the same construction, the vector field $\xi$ on $X_{S}$ induces an endomorphism of $M\left(X_{S}\right)$, and therefore of the vector space $\mathrm{HP}_{0}\left(X_{S}\right)$. In this case, since we do not assume that $\xi$ integrates to an honest action of $\mathbf{C}^{\times}$, we do not know a priori that the endomorphism is semisimple. The following result says that it is, and that the induced gradings on $L_{S, s} \cong \operatorname{HP}_{0}\left(X_{S}\right)$ agree up to a shift.

Theorem 5.1 The endomorphism of $\mathrm{HP}_{0}\left(X_{S}\right)$ induced by $\xi$ is semisimple, and the canonical vector space isomorphism $L_{S, s} \rightarrow \mathrm{HP}_{0}\left(X_{S}\right)[n \operatorname{dim} S / 2]$ respects the weight gradings.

In many cases, including hypertoric and Nakajima quiver varieties, the local systems $L_{S} \cong K_{S}$ are trivial. This allows us to conclude the following two corollaries.

Corollary 5.2 If the local systems $\left\{K_{S}\right\}$ are trivial, then there is an isomorphism of weakly $\mathbf{C}^{\times}$-equivariant D-modules

$$
M(X) \cong \sum_{S} \mathrm{IC}\left(S ; L_{S}\right) \cong \sum_{S} \operatorname{IC}\left(S ; \Omega_{S}\right) \otimes \operatorname{HP}_{0}\left(X_{S}\right)[n \operatorname{dim} S / 2]
$$

where the grading on $\mathrm{HP}_{0}\left(X_{S}\right)$ is induced by the (possibly infinitesimal) action of $\mathbf{C}^{\times}$on $X_{S}$.

Define $P_{X}(x, y)$ to be the Poincaré polynomial of $\mathrm{HP}_{*}(X)$, where $x$ records homological degree and $y$ records weights for the $\mathbf{C}^{\times}$-action. Note that weights can be both positive and negative, so $P_{X}(x, y)$ is a polynomial in $x, y$, and $y^{-1}$. For each leaf $S$, let $Q_{\bar{S}}(x)$ be the intersection cohomology Poincaré polynomial of $\bar{S}$, that is, $Q_{\bar{S}}(x):=\sum \operatorname{dim} \operatorname{IH}^{k}(\bar{S} ; \mathbf{C}) x^{k}$.

Corollary 5.3 If the local systems $\left\{K_{S}\right\}$ are trivial, then

$$
P_{X}(x, y)=\sum_{S} x^{\operatorname{dim} S} y^{-n \operatorname{dim} S / 2} Q_{\bar{S}}\left(x^{-1}\right) P_{X_{S}}(0, y)
$$

Proof: Let $\pi$ be the map from $X$ to a point. Then the corollary follows from Corollary 5.2 and the fact that $\mathrm{H}^{-k}\left(\pi_{*} \operatorname{IC}\left(S ; \Omega_{S}\right)\right) \cong \operatorname{IH}^{\operatorname{dim} S-k}(\bar{S} ; \mathbf{C})$. 
Remark 5.4 The first author has conjectured that, if $n=2$ and $X^{!}$is symplectic dual to $X$ in the sense of [BLPW, 10.15], then $P_{X}(0, y)=Q_{X !}(y)$ [Pro14, 3.4]. Thus, if each $K_{S}$ is trivial, each slice $X_{S}$ has a symplectic dual, and the aforementioned conjecture holds, then Corollary 5.3 allows us to express $P_{X}(x, y)$ entirely in terms of intersection cohomology Poincaré polynomials 6

In the next two sections, we will apply this result to compute $P_{X}(x, y)$ when $X$ is a unimodular hypertoric cone or a type A S3-variety. In both of these cases, the leaf closures, the slices, and their symplectic duals are varieties of the same type BLPW, 10.4, 10.8, 10.16, 10.18, and 10.19]; the local systems are trivial; and we know how to compute their intersection cohomology Poincaré polynomials. In the former case we obtain $h$-polynomials of the broken circuit complexes of matroids, and in the latter case we obtain Kostka polynomials. The conjecture about symplectic duals is proved for hypertoric varieties but not for S3-varieties, thus the computations in Section 7 are conditional on this unproved statement.

To prove Theorem 5.1, we need the following result. Let $U_{s}$ be a contractible open neighborhood of $s$ in the analytic topology.

Proposition 5.5 Every analytic Poisson vector field on $U_{s}$ is Hamiltonian, and so is any algebraic Poisson vector field on the formal completion $\hat{X}_{s}$.

Proof: By normality of $X$, it is enough to prove each statement on the regular locus. On a smooth symplectic manifold, Poisson vector fields correspond to closed one-forms and Hamiltonian vector fields correspond to exact one-forms. Thus, we need to show that the global sections of the de Rham complex has vanishing first cohomology on the regular locus. For the analytic statement, it suffices to show that the topological cohomology of $U_{s}^{\text {reg }}$ vanishes, since in this case every closed one-form is the differential of a smooth function, and if the one-form is analytic, the same must be true of the function.

We begin by observing that $U_{s}^{\text {reg }} \cong \rho^{-1}\left(U_{s}^{\text {reg }}\right)$. By [Kal06, 2.12], $\mathrm{H}^{1}\left(\rho^{-1}\left(U_{s}\right) ; \mathbf{C}\right)=0$, so we need to show that passing to the preimage of the regular locus does not introduce any cohomology in degree 1 . Since $U_{s} \backslash U_{s}^{\text {reg }}$ has complex codimension at least one, hence real codimension at least two, every loop in $U_{s}$ can be homotoped to $U_{s}^{\text {reg }}$, so the map $\pi_{1}\left(U_{s}^{\text {reg }}\right) \rightarrow$ $\pi_{1}\left(U_{s}\right)$ is surjective. Consider the stratification

$$
\rho^{-1}\left(U_{s} \backslash U_{s}^{\mathrm{reg}}\right)=\bigsqcup_{S^{\prime}} \rho^{-1}\left(U_{s} \cap S^{\prime}\right)
$$

where $S^{\prime}$ ranges over all symplectic leaves of $X$ whose closure contains $S$ other than the open leaf. Suppose $S^{\prime}$ is such a leaf. By the semismallness property [Kal06, 2.11], the codimension of $\rho^{-1}\left(U_{s} \cap S^{\prime}\right)$ is at least half the codimension of $S^{\prime}$. If the codimension of $S^{\prime}$ is

\footnotetext{
${ }^{6}$ As explained in Pro14, the full version of the conjecture [Pro14, 3.4] applied to a slice $X_{S}$ with a symplectic dual would imply that $\operatorname{dim} \operatorname{HP}_{0}\left(X_{S}\right)=\operatorname{rk} K_{S}$, thus the hypothesis of Theorem 4.1 would be satisfied.
} 
at least four, then $\rho^{-1}\left(U_{s} \cap S^{\prime}\right)$ therefore has codimension at least two, hence real codimension four. The fundamental group of a smooth manifold is unchanged by removing a locus of real codimension greater than two (since homotopies of loops can be pushed off this locus). Therefore the fundamental group of $U_{s}$ is unchanged by removing the union of $\rho^{-1}\left(U_{s} \cap S^{\prime}\right)$ over all leaves $S^{\prime}$ of codimension at least four. Next, if $S^{\prime}$ has codimension two, then the singularity at $s^{\prime} \in S^{\prime}$ is of Kleinian type, and hence in a small enough neighborhood $U_{s^{\prime}}$ of $s^{\prime}$, the fundamental group $\pi_{1}\left(\rho^{-1}\left(U_{s^{\prime}}\right)\right)$ is a finite subgroup of $\mathrm{SL}_{2}(\mathbf{C})$. Therefore, the kernel of the map $\pi_{1}\left(\rho^{-1}\left(U_{s} \backslash S^{\prime}\right)\right) \rightarrow \pi_{1}\left(\rho^{-1}\left(U_{s}\right)\right)$ is generated by this finite subgroup of $\mathrm{SL}_{2}(\mathbf{C})$. We conclude that the surjection $\pi_{1}\left(\rho^{-1}\left(U_{s}^{\text {reg }}\right)\right) \rightarrow \pi_{1}\left(\rho^{-1}\left(U_{s}\right)\right)$ is generated by elements of finite order, and hence this surjection descends to an isomorphism on homology

$$
0=\mathrm{H}_{1}\left(\rho^{-1}\left(U_{s}^{\mathrm{reg}}\right), \mathbf{C}\right) \stackrel{\cong}{\longrightarrow} \mathrm{H}_{1}\left(\rho^{-1}\left(U_{s}\right), \mathbf{C}\right) .
$$

Dualizing, we obtain the desired result.

For the statement about the formal completion, we follow [ES, §4.4]. Let $V:=U \backslash U_{s}^{\text {reg }}$ be the singular locus. By Hartshorne's theorem Har72, Har75, the de Rham hypercohomology of the formal completion $\hat{\tilde{X}}_{\rho^{-1}(s)}$ equals the topological cohomology of the fiber $\rho^{-1}(s)$, which also equals the topological cohomology of $\rho^{-1}\left(U_{s}\right)$. Then, as in [ES, (4.40)], the MayerVietoris sequences for the triples $\left(\tilde{X}, \tilde{X} \backslash \rho^{-1}(V), \hat{\tilde{X}}_{\rho^{-1}(s)}\right)$ and $\left(\tilde{X}, \tilde{X} \backslash \rho^{-1}(V), \rho^{-1}\left(U_{s}\right)\right)$ are isomorphic. Since the intersections of the second two open subsets of $\tilde{X}$ are $\rho^{-1}\left(\hat{X}_{s}^{\text {reg }}\right) \cong \hat{X}_{s}^{\text {reg }}$ and $\rho^{-1}\left(U_{s}^{\text {reg }}\right) \cong U_{s}^{\text {reg }}$, respectively, we may take hypercohomology of the de Rham complex to conclude that $\mathbf{H}_{D R}^{1}\left(\hat{X}_{s}^{\mathrm{reg}}\right) \cong \mathbf{H}_{D R}^{1}\left(U_{s}^{\mathrm{reg}}\right)$. The latter, by Grothendieck's theorem, is equal to the topological cohomology, which we showed is zero.

To conclude, we need to compare the hypercohomology of the de Rham complex with the cohomology of global sections of the de Rham complex. The spectral sequence computing hypercohomology degenerates in degree one on the second page, yielding an isomorphism $\mathbf{H}_{D R}^{1}\left(\hat{X}_{s}^{\text {reg }}\right) \cong H^{1}\left(\Gamma\left(\Omega_{\hat{X}_{s}^{\text {reg }}}\right)\right) \oplus\left(R^{1} \Gamma\right)\left(\mathcal{O}_{\hat{X}_{s}^{\text {reg }}}\right)$. Hence both summands are zero.

Proof of Theorem 5.1: Passing to a formal neighborhood of $s$, we obtain the DarbouxWeinstein decomposition $\hat{X}_{s} \cong \hat{S}_{s} \hat{\times} X_{S}$. Then we can view $\xi$ as a vector field on $\hat{X}_{s}$ parallel to the $X_{S}$ factor everywhere, and set $\xi^{\prime}:=\operatorname{Eu}_{\hat{S}_{s}}+\xi$. Letting $\pi$ be the Poisson bivector on $X$ and hence on $\hat{X}_{s}$, we have $L_{\xi^{\prime}} \pi=-n \pi=L_{\mathrm{Eu}} \pi$. This implies that $\xi^{\prime}-\mathrm{Eu}$ is Poisson, and therefore Hamiltonian by Proposition 5.5. Thus the endomorphisms of $M\left(\hat{X}_{s}\right) \cong M\left(\hat{S}_{s}\right) \otimes M\left(X_{S}\right)$ induced by $\mathrm{Eu}$ and $\xi^{\prime}$ are equal.

The endomorphism induced by $\mathrm{Eu}$ is responsible for the grading on $L_{S, s}$, and the endomorphism of $M\left(X_{S}\right)$ induced by $\xi$ is responsible for the grading on $\operatorname{HP}_{0}\left(X_{S}\right)$. To prove the theorem, we need to show that the endomorphism of $M\left(\hat{S}_{s}\right)$ induced by $\mathrm{Eu}_{\hat{S}_{s}}$ is multiplication by $-n \operatorname{dim} S / 2$. To see this last fact, note that $M\left(\hat{S}_{s}\right) \cong \Omega_{\hat{S}_{s}}$ via the map that sends the 
canonical generator to $\omega^{\operatorname{dim} S / 2}$, where $\omega$ is the symplectic form on $\hat{S}_{s}$. Since the Lie derivative map from vector fields to differential operators is an antihomomorphism, we have

$$
\mathrm{Eu}_{\hat{S}_{s}} \cdot \omega^{\operatorname{dim} S / 2}=-L_{\mathrm{Eu}_{\hat{S}_{s}}} \omega^{\operatorname{dim} S / 2}=-(n \operatorname{dim} S / 2) \omega^{\operatorname{dim} S / 2},
$$

by our assumption that the Poisson bracket on $X$, and hence on $S$, has weight $-n$.

\section{The hypertoric case}

In this section we compute the polynomial $P_{X(\mathcal{A})}(x, y)$ for a coloop-free, unimodular, rational, central hyperplane arrangement $\mathcal{A}$ with $\ell$ hyperplanes. We use the action of $\mathbf{C}^{\times}$described in [Pro14, §2], for which the symplectic form on the resolution has weight $n=2$.

Denham [Den01, §3] defines a polynomial $\Phi_{\mathcal{A}}\left(x, y, b_{1}, \ldots, b_{\ell}\right)$ whose coefficients are the dimensions of certain eigenspaces (determined by the $b$ exponents) of "combinatorial Laplacian" operators on certain vector spaces (determined by the $x$ and $y$ exponents). We will identify all of the $b$ variables to obtain a 3 -variable polynomial $\Phi_{\mathcal{A}}(x, y, b)$. This is an enrichment of the Tutte polynomial in the sense that $\Phi_{\mathcal{A}}(x-1, y-1,1)=T_{\mathcal{A}}(x, y)$ [Den01, 23(2)].

Theorem 6.1 $P_{X(\mathcal{A})}(x, y)=y^{-2 \operatorname{rk} \mathcal{A}} \Phi_{\mathcal{A}}\left(x^{2}+1, y^{-2}+1, y^{2}\right)$.

Proof: As stated in Example 4.6, the symplectic leaves of $X(\mathcal{A})$ are indexed by coloopfree flats of $\mathcal{A}$, and the leaf indexed by $F$ has a formal slice that is isomorphic to a formal neighborhood of the cone point of $X\left(\mathcal{A}_{F}\right)$. Furthermore, the closure of the leaf is isomorphic to $X\left(\mathcal{A}^{F}\right)$, where $\mathcal{A}^{F}$ is the restriction of $\mathcal{A}$ to $F$ [PW07, $\left.\S 2\right]$.

Let $T_{\mathcal{A}}(x, y)$ be the Tutte polynomial of $\mathcal{A}$. By [PW07, 4.3 and 5.5], we have

$$
Q_{X(\mathcal{A})}(x)=h_{\mathcal{A}}^{\mathrm{br}}\left(x^{2}\right)=x^{2 \operatorname{rk} \mathcal{A}} T_{\mathcal{A}}\left(x^{-2}, 0\right)
$$

Applying this to the restricted arrangement $\mathcal{A}^{F}$, we obtain

$$
Q_{X\left(\mathcal{A}^{F}\right)}(x)=x^{2 \operatorname{crk} F} T_{\mathcal{A}^{F}}\left(x^{-2}, 0\right)
$$

where $\operatorname{crk} F=\operatorname{rk} \mathcal{A}-\operatorname{rk} F$. By [Pro14, 3.1], we have

$$
P_{X(\mathcal{A})}(0, y)=Q_{X\left(\mathcal{A}^{\vee}\right)}(y)=y^{2 \operatorname{rk} \mathcal{A}^{\vee}} T_{\mathcal{A}^{\vee}}\left(y^{-2}, 0\right)=y^{2|\mathcal{A}|-2 \operatorname{rk} \mathcal{A}} T_{\mathcal{A}}\left(0, y^{-2}\right)
$$

where $\mathcal{A}^{\vee}$ is the Gale dual of $\mathcal{A}$. Applying this to the localized arrangement $\mathcal{A}_{F}$, we obtain

$$
P_{X\left(\mathcal{A}_{F}\right)}(0, y)=y^{2|F|-2 \operatorname{rk} F} T_{\mathcal{A}_{F}}\left(0, y^{-2}\right)
$$


Applying Corollary 5.3, we have

$$
\begin{aligned}
P_{X(\mathcal{A})}(x, y) & =\sum_{F} x^{2 \operatorname{crk} F} y^{-2 \operatorname{crk} F} x^{-2 \operatorname{crk} F} T_{\mathcal{A}^{F}}\left(x^{2}, 0\right) y^{2|F|-2 \operatorname{rk} F} T_{\mathcal{A}_{F}}\left(0, y^{-2}\right) \\
& =y^{-2 \operatorname{rk} \mathcal{A}} \sum_{F} y^{2|F|} T_{\mathcal{A}^{F}}\left(x^{2}, 0\right) T_{\mathcal{A}_{F}}\left(0, y^{-2}\right) .
\end{aligned}
$$

Let $\chi_{\mathcal{A}}(x)=(-1)^{\mathrm{rk} \mathcal{A}} T_{\mathcal{A}}(1-x, 0)$ be the characteristic polynomial of $\mathcal{A}$. By the first equation in Den01, §3.1], we have

$$
\begin{aligned}
\Phi_{\mathcal{A}}(x, y, b) & =\sum_{F}(-1)^{\mathrm{rk} \mathcal{A}-|F|} \chi_{\mathcal{A}^{F}}(-x) \chi_{\left(\mathcal{A}_{F}\right)^{\vee}}(-y) b^{|F|} \\
& =\sum_{F}(-1)^{\mathrm{rk} \mathcal{A}-|F|}(-1)^{\mathrm{rk} \mathcal{A}^{F}}(-1)^{\mathrm{rk}\left(\mathcal{A}_{F}\right)^{\vee}} T_{\mathcal{A}^{F}}(x-1,0) T_{\left(\mathcal{A}_{F}\right)^{\vee}}(y-1,0) b^{|F|} \\
& =\sum_{F} T_{\mathcal{A}^{F}}(x-1,0) T_{\left(\mathcal{A}_{F}\right)^{\vee}}(y-1,0) b^{|F|} \\
& =\sum_{F} T_{\mathcal{A}^{F}}(x-1,0) T_{\mathcal{A}_{F}}(0, y-1) b^{|F|} .
\end{aligned}
$$

Thus

$$
y^{-2 \operatorname{rk} \mathcal{A}} \Phi_{\mathcal{A}}\left(x^{2}+1, y^{-2}+1, y^{2}\right)=y^{-2 \operatorname{rk} \mathcal{A}} \sum_{F} T_{\mathcal{A}^{F}}\left(x^{2}, 0\right) T_{\mathcal{A}_{F}}\left(0, y^{-2}\right) y^{2|F|}=P_{X(\mathcal{A})}(x, y),
$$

and the theorem is proved.

Remark 6.2 Specializing at $y=1$, we obtain the equation

$$
P_{X(\mathcal{A})}(x, 1)=\Phi_{\mathcal{A}}\left(x^{2}+1,2,1\right)=T_{\mathcal{A}}\left(x^{2}, 1\right)=x^{2 \operatorname{rk} \mathcal{A}_{\mathcal{A}}} h\left(x^{-2}\right)
$$

matching the known formula for the Betti numbers of a conical symplectic resolution of $X(\mathcal{A})$ given in [HS02, 1.2] and [PW07, 3.5 and 5.5].

\section{The case of S3-varieties in type A}

Let $\lambda$ and $\mu$ be partitions of the same positive integer $r$. Let $\mathcal{O}_{\lambda}$ be the nilpotent coadjoint orbit in $\mathfrak{s l}_{r}^{*}$ whose Jordan blocks have sizes given by the parts of $\lambda \sqrt{7}$ then $\lambda \geq \mu$ in the dominance order if and only if $\mathcal{O}_{\mu}$ is contained in the closure of $\mathcal{O}_{\lambda}$. In this case, let $X_{\lambda \mu}$ be the normal slice to $\mathcal{O}_{\mu}$ inside of the closure of $\mathcal{O}_{\lambda}$. This space is sometimes called an

\footnotetext{
${ }^{7}$ More precisely, the elements of the image of this orbit under the Killing form isomorphism $\mathfrak{s l}_{r}^{*} \rightarrow \mathfrak{s l}_{r}$ have this Jordan decomposition.
} 
S3-variety, after Slodowy, Spaltenstein, and Springer [Web11, BLPW]. The variety $X_{\lambda \mu}$ is a Nakajima quiver variety for a finite type A quiver, and conversely any such variety is an S3-variety [Maf05]; in particular, $X_{\lambda \mu}$ admits a projective symplectic resolution (the fibers are known as Spaltenstein varieties), and the local systems associated to the symplectic leaves are trivial. We equip these varieties with the standard action of $\mathbf{C}^{\times}$with the property that the Poisson bracket is homogeneous of weight -2 . The symplectic leaves of $X_{\lambda \mu}$ are indexed by the poset $[\mu, \lambda]$. For any $\nu \in[\mu, \lambda]$, the closure of the leaf $S_{\nu}$ is isomorphic to $X_{\nu \mu}$, and the normal slice to $S_{\nu}$ is isomorphic to $X_{\lambda \nu}$.

Let $n_{\lambda}=\sum_{i}(i-1) \lambda_{i}$, so that $\operatorname{dim} X_{\lambda \mu}=2\left(n_{\mu}-n_{\lambda}\right)$. A theorem of Lusztig Lus81, Theorem 2] says that

$$
Q_{X_{\lambda \mu}}(x)=x^{2\left(n_{\mu}-n_{\lambda}\right)} K_{\lambda \mu}\left(x^{-2}\right),
$$

where $K_{\lambda \mu}(t)$ is the Kostka polynomial associated to $\lambda$ and $\mu$. We will assume that the conjecture [Pro14, 3.4] holds; we have $X_{\lambda \mu}^{!}=X_{\mu^{t} \lambda^{t}}$, so the explicit statement of the conjecture in this case is that

$$
P_{X_{\lambda \mu}}(0, y)=Q_{X_{\mu^{t} \lambda^{t}}}(y)=y^{2\left(n_{\lambda^{t}}-n_{\mu^{t}}\right)} K_{\mu^{t} \lambda^{t}}\left(y^{-2}\right)
$$

Proposition 7.1 If Equation (17.2) holds for all type A S3-varieties, then

$$
P_{X_{\lambda \mu}}(x, y)=y^{2\left(n_{\lambda^{t}}-n_{\mu}\right)} \sum_{\nu \in[\mu, \lambda]} y^{2\left(n_{\nu}-n_{\nu^{t}}\right)} K_{\nu \mu}\left(x^{2}\right) K_{\nu^{t} \lambda^{t}}\left(y^{-2}\right) .
$$

Proof: For all $\nu \in[\mu, \lambda]$, Equation (17.2) tells us that

$$
\operatorname{dim} \operatorname{HP}_{0}\left(X_{\lambda \nu}\right)=P_{X_{\lambda \nu}}(0,1)=K_{\nu^{t} \lambda^{t}}(1)
$$

which is in turn equal to the rank of the local system $K_{S_{\nu}}$ [BM83, 3.5(b)], thus the hypothesis of Theorem 4.1 is satisfied. Then by Corollary 5.3, we have

$$
\begin{aligned}
P_{X_{\lambda \mu}}(x, y) & =\sum_{\nu \in[\mu, \lambda]} x^{2\left(n_{\mu}-n_{\nu}\right)} y^{-2\left(n_{\mu}-n_{\nu}\right)} x^{2\left(n_{\nu}-n_{\mu}\right)} K_{\nu \mu}\left(x^{2}\right) y^{2\left(n_{\lambda^{t}}-n_{\nu^{t}}\right)} K_{\nu^{t} \lambda^{t}}\left(y^{-2}\right) \\
& =y^{2\left(n_{\lambda^{t}}-n_{\mu}\right)} \sum_{\nu \in[\mu, \lambda]} y^{2\left(n_{\nu}-n_{\nu^{t}}\right)} K_{\nu \mu}\left(x^{2}\right) K_{\nu^{t} \lambda^{t}}\left(y^{-2}\right) .
\end{aligned}
$$

This completes the proof. 


\section{The case of the nilpotent cone in general type}

Let $\mathfrak{g}$ be any semisimple Lie algebra, and let $X \subseteq \mathfrak{g}^{*}$ be the nilpotent cone. As before, one can consider coadjoint orbits in $X$ and slices to one inside the closure of another; however, these do not admit symplectic resolutions in general, and even when they do, the assumptions of Theorem 4.1 are not known to be satisfied. Here we consider only the case of $X$ itself, where Theorem 4.1] is known to hold for the Springer resolution $T^{*} \mathcal{B} \rightarrow X$ [ES10b], where $\mathcal{B}$ is the flag variety 8 If $\mathfrak{g}$ is not of type $A$, then the hypothesis of Corollary 5.3 fails, so we have no direct way of using that result to compute $P_{X}(x, y)$. However, we will conjecture a formula for $P_{X}(x, y)$ based on the type A case and a suggestion of G. Lusztig and P. Etingof.

\subsection{Generalized Kostka polynomials}

Let $W$ be the Weyl group of $\mathfrak{g}$. Springer theory tells us that $T$ is equipped with an action of $W$, and that for every irreducible representation $\chi$ of $W$, we may associate a nilpotent coadjoint orbit $\mathcal{O}_{\mathfrak{g}, \chi}$ and an irreducible local system $M_{\mathfrak{g}, \chi}$ on $\mathcal{O}_{\mathfrak{g}, \chi}$ such that

$$
M \cong T \cong \bigoplus_{\mathfrak{g}, \chi} \operatorname{IC}\left(\mathcal{O}_{\mathfrak{g}, \chi} ; M_{\mathfrak{g}, \chi}\right) \otimes \chi
$$

as a $W$-equivariant D-module. By pushing forward to a point and taking cohomology, we obtain an action of $W$ on $\mathrm{H}^{*}\left(T^{*} \mathcal{B} ; \mathbf{C}\right)=H^{*}(\mathcal{B} ; \mathbf{C})$ which is isomorphic (after forgetting the grading) to the regular representation.

For each $\chi$ of $W$, let

$$
K_{\mathfrak{g}, \chi}(t):=\sum_{i \geq 0} t^{i} \operatorname{dim} \operatorname{Hom}_{W}\left(\chi, \mathrm{H}^{2 \operatorname{dim} \mathcal{B}-2 i}(\mathcal{B} ; \mathbf{C})\right)
$$

We call $K_{\mathfrak{g}, \chi}(t)$ a generalized Kostka polynomial, motivated by the following well-known proposition.

Proposition 8.1 For any $\mathfrak{g}$ and any representation $\chi$ of $W$, we have

$$
K_{\mathfrak{g}, \chi}\left(t^{2}\right)=\sum_{i \geq 0} t^{i} \operatorname{dim} \operatorname{IH}^{\operatorname{dim} \mathcal{O}_{\mathfrak{g}, \chi}-i}\left(\overline{\mathcal{O}}_{\mathfrak{g}, \chi} ; M_{\mathfrak{g}, \chi}\right)
$$

If $\mathfrak{g}=\mathfrak{s l}_{r}, \chi$ is an irreducible representation of $S_{r}$, and $\nu$ is the partition of $r$ with the property that $\mathcal{O}_{\mathfrak{g}, \chi}=\mathcal{O}_{\nu}$, then $K_{\mathfrak{g}, \chi}(t)=K_{\nu\left(1^{r}\right)}(t)$.

Proof: The first statement follows immediately from pushing Equation (8.1) forward to a point and taking cohomology. To obtain the second statement from the first, we use Equation

\footnotetext{
${ }^{8}$ In fact, in [ES10b] the conclusion $M \cong T$ of Theorem 4.1 is proved first, and then the hypothesis follows.
} 
(7.1) (for $\mu=\left(1^{r}\right)$ and $\lambda=\nu$ ), along with the fact that, in type $A$, all the local systems $M_{\mathfrak{g}, \chi}$ are trivial.

Remark 8.2 By Poincaré duality, $\operatorname{Hom}_{W}\left(\chi, H^{2 i}(\mathcal{B} ; \mathbf{C})\right) \cong \operatorname{Hom}_{W}\left(\chi \otimes \sigma, H^{2 \operatorname{dim} \mathcal{B}-2 i}(\mathcal{B} ; \mathbf{C})\right)$, thus we also have

$$
K_{\mathfrak{g}, \chi}(t)=\sum_{i \geq 0} t^{i} \operatorname{dim} \operatorname{Hom}_{W}\left(\chi \otimes \sigma, \mathrm{H}^{2 i}(\mathcal{B} ; \mathbf{C})\right)
$$

Remark 8.3 Note that $\mathrm{H}^{*}(\mathcal{B} ; \mathbf{C})$ is canonically isomorphic as a $W$-equivariant graded algebra to the coinvariant algebra $\mathbf{C}[\mathfrak{h}] /\left(\mathbf{C}[\mathfrak{h}]_{+}^{W}\right)$, where $\mathbf{C}[\mathfrak{h}]_{+} \subset \mathbf{C}[\mathfrak{h}]$ is the augmentation ideal and $\mathfrak{h}^{*} \subset \mathbf{C}[\mathfrak{h}]$ sits in degree 2 .

\subsection{The conjecture}

Since the summand $\operatorname{IC}\left(\mathcal{O}_{\mathfrak{g}, \chi} ; M_{\mathfrak{g}, \chi}\right)$ of $M$ is simple, the weak $\mathbf{C}^{\times}$-equivariant structure on $M$ induces a grading on the multiplicity space $\chi$. Let $h(\chi ; t)$ be the Hilbert series for this grading.

Conjecture 8.4 For each irreducible representation $\chi$ of $W$, we have

$$
h(\chi ; y)=K_{\mathfrak{g}, \chi}\left(y^{-2}\right)
$$

and therefore

$$
P_{X}(x, y)=\sum_{\chi \in \operatorname{Irrep}(W)} K_{\mathfrak{g}, \chi}\left(x^{2}\right) K_{\mathfrak{g}, \chi}\left(y^{-2}\right) .
$$

Remark 8.5 Conjecture 8.4 holds at the specialization $y=1$ by the fact that $H^{*}(\mathcal{B} ; \mathbf{C})$ is isomorphic to the regular representation of $W$.

Remark 8.6 If $M_{\mathfrak{g}, \chi}$ is trivial, then $\operatorname{IH}^{0}\left(\overline{\mathcal{O}}_{\mathfrak{g}, \chi} ; M_{\mathfrak{g}, \chi}\right) \cong \mathbf{C}$, thus Proposition 8.1 tells us that the top degree of $K_{\mathfrak{g}, \chi}\left(x^{2}\right)$ is equal to the dimension of $\mathcal{O}_{\mathfrak{g}, \chi}$. Similarly, the bottom degree of $K_{\mathfrak{g}, \chi}\left(y^{-2}\right)$ is equal to $-\operatorname{dim} \mathcal{O}_{\mathfrak{g}, \chi}$, which is what the bottom degree of $h(\chi)$ should be according to Theorem 5.1 .

Remark 8.7 By Theorem 5.1, Conjecture 8.4 implies that, for each nilpotent orbit $S$,

$$
P_{X_{S}}(0, y)=\sum y^{\operatorname{dim} \mathcal{O}_{\mathfrak{g}, \chi}} K_{\mathfrak{g}, \chi}\left(y^{-2}\right)
$$

where the sum is taken over all $\chi$ such that $\mathcal{O}_{\mathfrak{g}, \chi}=S$. If there is only one such $\chi$ (and hence $M_{\mathfrak{g}, \chi}$ is trivial), then Conjecture 8.4 for $\chi$ is equivalent to Equation (8.2) for $S$. 
Example 8.8 In the case where $\chi=$ triv, which corresponds to the trivial local system on the open orbit, Equation (8.2) says that $h(\operatorname{triv} ; y)=y^{-\operatorname{dim} X}$. On the other hand, if $\chi=\sigma$, which corresponds to the cone point, it says $h(\sigma ; y)=1$. These conclusions both agree with Theorem [5.1, since in both cases the Poisson homology of the slice is one-dimensional and concentrated in degree zero.

Proposition 8.9 If $\mathfrak{g}=\mathfrak{s l}_{r}$, then the first formula of Conjecture 8.4 agrees with Equation (7.2) and the second with Proposition 7.1.

Proof: If $\mathcal{O}_{\mathfrak{g}, \chi}=\mathcal{O}_{\nu}$, then Equation (7.2) with $\lambda=(r)$ and $\mu=\nu$ tells us that

$$
P_{X_{(r) \nu}}(0, y)=y^{2\left(n_{\left(1^{r}\right)}-n_{\nu^{t}}\right)} K_{\nu^{t}\left(1^{r}\right)}\left(y^{-2}\right)=y^{\operatorname{dim} \mathcal{O}_{\nu^{t}}} K_{\nu^{t}\left(1^{r}\right)}\left(y^{-2}\right)=y^{\operatorname{dim} \mathcal{O}_{\mathfrak{g}, \chi \otimes \sigma}} K_{\mathfrak{g}, \chi \otimes \sigma}\left(y^{-2}\right) .
$$

On the other hand, the first formula of Conjecture 8.4 is equivalent to Equation (8.2), which says that

$$
P_{X_{(r) \nu}}(0, y)=y^{\operatorname{dim} \mathcal{O}_{\mathfrak{g}, \chi}} K_{\mathfrak{g}, \chi}\left(y^{-2}\right) .
$$

Thus we need to prove the following identity:

$$
K_{\mathfrak{g}, \chi}\left(t^{2}\right)=t^{\operatorname{dim} \mathcal{O}_{\mathfrak{g}, \chi}-\operatorname{dim} \mathcal{O}_{\mathfrak{g}, \chi \otimes \sigma}} K_{\mathfrak{g}, \chi \otimes \sigma}\left(t^{2}\right)
$$

Using Poincaré duality (Remark 8.2) and the fact that $\operatorname{dim} \mathcal{O}_{\mathfrak{g}, \chi}=r(r-1)-n_{\nu}$, this identity reduces to the following palindromic property of $K_{\mathfrak{g}, \chi}\left(t^{2}\right)$ :

$$
K_{\mathfrak{g}, \chi}\left(t^{2}\right)=t^{n_{\nu}-n_{\nu}+r(r-1)} K_{\mathfrak{g}, \chi}\left(t^{-2}\right)
$$

This follows from [BL78, Propositions A and B, (1)], and (as explained there) is originally due to Steinberg [Ste51].

Remark 8.10 As pointed out by G. Lusztig, it is possible to generalize (8.3) to arbitrary irreducible types. For any irreducible representation $\chi$ of $W$, let $\chi^{s}$ denote the unique special representation in the same two-sided cell as $\chi$. In [BL78], there is an involution $i$ defined on the set of irreducible representations of $W$, which is the identity except for six irreducible representations in types $E_{7}$ and $E_{8}$, called "exceptional" ones, which are exactly the representations for which $K_{\mathfrak{g}, \chi}(t)$ is not palindromic.

Lusztig pointed out that, combining [BL78, Propositions A and B] with the determinant of [Lus84, 5.12.2], and comparing powers of $u$ in the latter, one can conclude the following identity (when $W$ is irreducible):

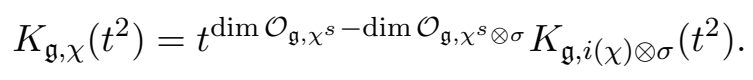


In type $A, i$ is trivial and $\chi=\chi^{s}$ for all $\chi$, thus we recover the identity in Equation (8.3).

Remark 8.11 Motivated by in part by symplectic duality [BLPW], we originally guessed the following formula for $h(\chi)$ :

$$
y^{-\operatorname{dim} \mathcal{O}_{\mathfrak{g}, \chi}+\operatorname{dim} \mathcal{O}_{\mathfrak{g}} L, \chi \otimes \sigma} K_{\mathfrak{g}, \chi \otimes \sigma}\left(y^{-2}\right) .
$$

(Here $\mathfrak{g}^{L}$ is the Langlands dual of $\mathfrak{g}$, whose Weyl group is canonically isomorphic to that of $\mathfrak{g}$.) This agrees with Conjecture 8.4 in all of the examples considered in this paper: types $A_{\ell}, B_{2}$, $C_{2}$, and $G_{2}$, and also for the subregular orbit in general (and, in the $B_{2}, C_{2}$, and $G_{2}$ cases, the Langlands duality is required for it to hold). However, as Lusztig pointed out, the formulas do not coincide in some cases, such as when $\chi$ is the (non-exceptional) 50-dimensional irreducible representation of $E_{8}$ for which $M_{\mathfrak{g}, \chi}$ is trivial; moreover, Remark 8.6 implies that our original guess was incorrect in this case.

\subsection{A proof of the conjecture along the subregular orbit}

In this subsection we verify Conjecture 8.4 when $\mathcal{O}_{\mathfrak{g}, \chi}$ is equal to the subregular orbit $R$. First suppose $\mathfrak{g}$ is simply laced; in this case, the only such representation is the reflection representation $\chi=\mathfrak{h}$.

Proposition 8.12 If $\mathfrak{g}$ is simply laced, then Conjecture 8.4 holds for $\chi=\mathfrak{h}$.

Proof: Since there is only one irreducible representation associated to the subregular orbit, Conjecture 8.4 for $\mathfrak{h}$ is equivalent to Equation (8.2) for $R$, which says

$$
P_{X_{R}}(0, y)=y^{\operatorname{dim} R} K_{\mathfrak{g}, \mathfrak{h}}\left(y^{-2}\right) .
$$

By Remarks 8.2 and 8.3 , since $\operatorname{codim} R=2$, we have

$$
y^{\operatorname{dim} R} K_{\mathfrak{g}, \mathfrak{h}}\left(y^{-2}\right)=y^{-2} h\left(\operatorname{Hom}_{W}\left(\mathfrak{h}, \mathbf{C}[\mathfrak{h}] /\left(\mathbf{C}[\mathfrak{h}]_{+}^{W}\right) ; y\right)\right.
$$

Consider the map from $\Phi: \mathbf{C}[\mathfrak{h}]^{W} \rightarrow \operatorname{Hom}_{W}\left(\mathfrak{h}, \mathbf{C}[\mathfrak{h}] /\left(\mathbf{C}[\mathfrak{h}]_{+}^{W}\right)\right)$ taking $f$ to $\Phi_{f}$, which is defined by the formula $\Phi_{f}(x):=\partial_{x}(f)$ for all $x \in \mathfrak{h}$. The restriction of $\Phi$ to the linear span of the fundamental invariants (the ring generators of $\mathbf{C}[\mathfrak{h}]^{W}$ ) is an isomorphism. Since $\Phi$ lowers degree by 2 , this implies that

$$
y^{\operatorname{dim} R} K_{\mathfrak{g}, \mathfrak{h}}\left(y^{-2}\right)=y^{-2} \sum_{i} y^{2 d_{i}-2}=\sum_{i} y^{2\left(d_{i}-2\right)},
$$


where $\left\{2 d_{i}\right\}$ are the degrees of the fundamental invariants 9 This indeed coincides with $P_{X_{R}}(0, y)$, as desired [Gre75, AL98.

In the non-simply laced case, let $\tilde{D}$ be the simply laced Dynkin diagram folding to the type of $\mathfrak{g}$, let $\tilde{W}$ be the corresponding Weyl group, and let $\tilde{\mathfrak{h}}$ be its reflection representation. As representations of $W$, we have $\tilde{\mathfrak{h}} \cong \mathfrak{h} \oplus \tau$ for some irreducible representation $\tau \neq \mathfrak{h}$ of $W$, and $\tau$ and $\mathfrak{h}$ are the only two irreducible representations lying over $R$. The slice $X_{R}$ is a Kleinian singularity of type $\tilde{D}$, and Theorem 5.1 tells us that $\operatorname{HP}_{0}\left(X_{R}\right)$ is isomorphic as a graded vector space to a fiber of the local system $L_{R}[-\operatorname{dim} R]$, where

$$
L_{R}=\left(M_{\mathfrak{g}, \mathfrak{h}} \otimes \mathfrak{h}\right) \oplus\left(M_{\mathfrak{g}, \tau} \otimes \tau\right)
$$

Proposition 8.13 If $\mathfrak{g}$ is not simply laced, then Conjecture 8.4 holds for $\chi=\mathfrak{h}$ and for $\chi=\tau$.

Proof: For $\chi=\mathfrak{h}$ or $\tau$, let $\operatorname{HP}_{0}\left(X_{R}\right)_{\chi} \subset \operatorname{HP}_{0}\left(X_{R}\right)$ be the summand corresponding to a fiber of the local system $M_{\mathfrak{g}, \mathfrak{h}}[-\operatorname{dim} R] \subset L_{R}[-\operatorname{dim} R]$. As in the proof of Proposition 8.12 , we need to show that the Hilbert series of $\operatorname{HP}_{0}\left(X_{R}\right)_{\chi}$ is equal to

$$
y^{-2} h\left(\operatorname{Hom}_{W}\left(\chi, \mathbf{C}[\mathfrak{h}] /\left(\mathbf{C}[\mathfrak{h}]_{+}^{W}\right)\right) ; y\right) .
$$

We first consider the case where $\chi=\mathfrak{h}$. The local system $M_{\mathfrak{g}, \mathfrak{h}}$ is trivial, so $\operatorname{HP}_{0}\left(X_{R}\right)_{\mathfrak{h}}$ is the part of $\operatorname{HP}_{0}\left(X_{R}\right)$ that is fixed by the action of $\pi_{1}(R)$. As in the proof of Proposition 8.12, Equation (8.5) simplifies to

$$
\sum_{i} y^{2\left(d_{i}-2\right)}
$$

where $\left\{2 d_{i}\right\}$ are the degrees of the fundamental invariants for the action of $W$ on $\mathbf{C}[\mathfrak{h}]$. (Note that these are a subset of the fundamental invariants for the action of $\tilde{W}$ on $\mathbf{C}[\tilde{\mathfrak{h}}]$.) We will check on a case-by-case basis that this is equal to the Hilbert series of $\operatorname{HP}_{0}\left(X_{R}\right)_{\mathfrak{h}}$. We will skip the case of $G_{2}$, since that will be treated separately in Proposition 8.15, In all other cases, $\pi_{1}(R) \cong \mathbf{Z} / 2$, and the action on $\mathrm{HP}_{0}\left(X_{R}\right)$ can be deduced from the explicit bases for the latter in $\left[\mathrm{EGP}^{+} 12, \S 5.1\right]$. It is straightforward to check that our formula is correct.

Next, consider the case $\chi=\tau$. In view of the above, we need to show that

$$
y^{-2} h\left(\operatorname{Hom}_{W}\left(\tau, \mathbf{C}[\mathfrak{h}] /\left(\mathbf{C}[\mathfrak{h}]_{+}^{W}\right)\right) ; y\right)=\sum_{i} y^{2\left(e_{i}-2\right)}
$$

where $\left\{2 e_{i}\right\}$ are the degrees of the fundamental invariants for the action of $\tilde{W}$ on $\mathbf{C}[\tilde{\mathfrak{h}}]$ that restrict to zero on $\mathfrak{h} \subseteq \tilde{\mathfrak{h}}$. To prove this, it is sufficient to show that there exists a graded

\footnotetext{
${ }^{9}$ The factor of 2 is there because $\mathfrak{h}^{*}$ sits in degree 2 .
} 
vector space isomorphism

$$
\operatorname{Hom}_{\tilde{W}}\left(\tilde{\mathfrak{h}}, \mathbf{C}[\tilde{\mathfrak{h}}] /\left(\mathbf{C}[\tilde{\mathfrak{h}}]_{+}^{\tilde{W}}\right)\right) \cong \operatorname{Hom}_{W}\left(\tilde{\mathfrak{h}}, \mathbf{C}[\mathfrak{h}] /\left(\mathbf{C}[\mathfrak{h}]_{+}^{W}\right)\right)
$$

The restriction map from $\mathbf{C}[\tilde{\mathfrak{h}}]$ to $\mathbf{C}[\mathfrak{h}]$ induces a natural map from the left-hand side to the right-hand side. Moreover, both sides have the same dimension (equal to $\operatorname{dim} \tilde{\mathfrak{h}}$ ), since the coinvariant algebras for $W$ and $\tilde{W}$ are the regular representations of $W$ and $\tilde{W}$, respectively. Therefore, it suffices to prove that the natural map is injective.

Equivalently, we need to show that, for every fundamental invariant $f \in \mathbf{C}[\tilde{\mathfrak{h}}]^{\tilde{W}}$ which restricts to zero on $\tilde{\mathfrak{h}}$, the corresponding homomorphism $\Phi_{f} \in \operatorname{Hom}_{\tilde{W}}\left(\tilde{\mathfrak{h}}, \mathbf{C}[\tilde{\mathfrak{h}}] /\left(\mathbf{C}[\tilde{\mathfrak{h}}]_{+}^{\tilde{W}}\right)\right)$ defined above restricts to a nonzero element of $\operatorname{Hom}_{W}\left(\tau, \mathbf{C}[\mathfrak{h}] /\left(\mathbf{C}[\mathfrak{h}]_{+}^{W}\right)\right)$. This is easy to verify explicitly in the case where $\mathfrak{g}$ is of type $B_{n}$ (so $\tilde{D}=A_{2 n-1}$ ), using the embedding $W\left(B_{n}\right) \hookrightarrow W\left(A_{2 n-1}\right) \cong S_{2 n-1}$, since then $\mathbf{C}[\tilde{\mathfrak{h}}]^{\tilde{W}}$ is the ring of symmetric polynomials (modulo linear symmetric polynomials). In the case $C_{n}, \tau$ is one-dimensional and $\tau \otimes \tau$ is trivial, thus $\mathfrak{h}^{\perp} \subseteq(\tilde{\mathfrak{h}})^{*}$ is one-dimensional. Then, the fundamental invariant $f$ of $\mathbf{C}[\tilde{\mathfrak{h}}]^{\tilde{W}}$ which restricts to zero in $\mathbf{C}[\mathfrak{h}]^{W}$ lies in $\left(\mathfrak{h}^{\perp}\right)$ but not in $\left(\mathfrak{h}^{\perp}\right)^{2}$. It follows that the corresponding element $\Phi_{f}$ indeed restricts to a nonzero element of $\operatorname{Hom}_{W}\left(\tau, \mathbf{C}[\mathfrak{h}] /\left(\mathbf{C}[\mathfrak{h}]_{+}^{W}\right)\right)$. In the case $F_{4}$, one can explicitly verify the statement.

\subsection{Proof of the conjecture for semisimple Lie algebras of rank at most 2}

Conjecture 8.4 is easy to verify for $\mathfrak{g}$ of type $A_{1}$ and $A_{2}$ by checking Equation (7.2) in low dimensions 10 In the two remaining examples, we prove the conjecture for $\mathfrak{g}$ of type $B_{2}$ and $G_{2}$, and therefore for all all $\mathfrak{g}$ of semisimple rank at most 2 .

Proposition 8.14 Conjecture 8.4 for $\mathfrak{g}$ of type $B_{2}\left(\mathfrak{g}=\mathfrak{s o}_{5}\right)$.

Proof: There are four nilpotent orbits: the zero orbit, the minimal orbit (of dimension four), the subregular orbit (of dimension six), and the open orbit (of dimension eight). Call these $\mathrm{O}_{0}, \mathrm{O}_{2}, \mathrm{O}_{4}, \mathrm{O}_{6}$, and $\mathrm{O}_{8}$, where $\mathrm{O}_{k}$ has dimension $k$. These orbits are all simply-connected except for $O_{6}$, which has fundamental group $\mathbf{Z} / 2 \mathbf{Z}$. Let $\Omega_{k}$ denote the rank-one trivial local system on $O_{k}$, and let $L_{6}$ be the nontrivial rank-one local system (with regular singularities) on $O_{6}$. The Weyl group is isomorphic to the dihedral group of order eight, which has five irreducible representations: triv, $\sigma, \tau, \tau \otimes \sigma$, and $\mathfrak{h}$. The Springer correspondence for $\mathfrak{g}$ takes the following form [Car93, §13.3].

\footnotetext{
${ }^{10}$ In fact, in these cases, the result also follows from Example 8.8 and Proposition 8.12
} 


\begin{tabular}{|c|c|}
\hline$\chi$ & $\left(\mathcal{O}_{\mathfrak{g}, \chi}, M_{\mathfrak{g}, \chi}\right)$ \\
\hline $\operatorname{triv}$ & $\left(O_{8}, \Omega_{8}\right)$ \\
\hline$\sigma$ & $\left(O_{0}, \Omega_{0}\right)$ \\
\hline$\tau$ & $\left(O_{6}, L_{6}\right)$ \\
\hline$\tau \otimes \sigma$ & $\left(O_{4}, \Omega_{4}\right)$ \\
\hline $\mathfrak{h}$ & $\left(O_{6}, \Omega_{6}\right)$ \\
\hline
\end{tabular}

The $W$-equivariant Poincaré polynomial of the coinvariant algebra is equal to

$$
1+\mathfrak{h} \cdot t^{2}+(\tau+\tau \otimes \sigma) \cdot t^{4}+\mathfrak{h} \cdot t^{6}+\sigma \cdot t^{8}
$$

therefore

$$
K_{\mathfrak{g}, \text { triv }}\left(t^{2}\right)=t^{8}, \quad K_{\mathfrak{g}, \sigma}\left(t^{2}\right)=1, \quad K_{\mathfrak{g}, \tau}\left(t^{2}\right)=t^{4}=K_{\mathfrak{g}, \tau \otimes \sigma}\left(t^{2}\right), \quad K_{\mathfrak{g}, \mathfrak{h}}\left(t^{2}\right)=t^{2}+t^{6}
$$

Thus, Conjecture 8.4 says that

$$
h(\operatorname{triv} ; y)=y^{-8}, \quad h(\sigma ; y)=1, \quad h(\tau ; y)=y^{-4}=h(\tau \otimes \sigma ; y), \quad h(\mathfrak{h} ; y)=y^{-2}+y^{-6} .
$$

All of the slices except the slice to $O_{6}$ have one-dimensional $\mathrm{HP}_{0}$, therefore the conjectural formulas for triv, $\sigma$, and $\tau \otimes \sigma$ follow from Theorem 5.1. Our table tells us that $\operatorname{IC}\left(O_{6} ; \Omega_{6}\right)$ appears in $M$ with multiplicity $2=\operatorname{dim} \mathfrak{h}$ and $\operatorname{IC}\left(O_{6} ; \Omega_{6}\right)$ appears in $M$ with multiplicity $1=\operatorname{dim} \sigma$. The slice to $O_{6}$ is a Kleinian singularity of type $A_{2}$, where a basis for $\mathrm{HP}_{0}$ is given by the images of $1, x y,(x y)^{2} \in \mathbf{C}[x, y]^{\mathbf{Z} / 3}$. Since the generator in top degree can be taken to be the square of the generator in middle degree, we see that the nontrivial local system $L_{6}$ must be in middle degree and the trivial one $\Omega_{6}$ must be in top and bottom degrees; this allows us to conclude that the formulas for $h(\sigma ; y)$ and $h(\mathfrak{h} ; y)$ are correct.

Proposition 8.15 Conjecture 8.4 holds for $\mathfrak{g}$ of type $G_{2}$.

Proof: There are five nilpotent orbits, call them $O_{0}, O_{6}, O_{8}, O_{10}$, and $O_{12}$ (again $\operatorname{dim} O_{k}=k$ ), and these are all simply-connected except for the subregular orbit $O_{10}$, which has fundamental group $S_{3}$ [Car93, p. 427]. Let $\Omega_{k}$ denote the trivial local system on $O_{k}$, and on $O_{10}$, let $L_{10}$ denote the local system corresponding to the reflection representation of the fundamental group $S_{3}$ (this is irreducible of rank two, with regular singularities).

Let $\tau$ be the irreducible one-dimensional representation of $W$ other than $\sigma$ (it is denoted by $\phi_{1,3}^{\prime}$ in [Car93, p. 412]). Then the Springer correspondence for $\mathfrak{g}$ takes the following form Car93, p. 427]. 


\begin{tabular}{|c|c|}
\hline$\chi$ & $\left(\mathcal{O}_{\mathfrak{g}, \chi}, M_{\mathfrak{g}, \chi}\right)$ \\
\hline 1 & $\left(O_{12}, \Omega_{12}\right)$ \\
\hline$\sigma$ & $\left(O_{0}, \Omega_{0}\right)$ \\
\hline$\tau$ & $\left(O_{10}, L_{10}\right)$ \\
\hline$\tau \otimes \sigma$ & $\left(O_{6}, \Omega_{6}\right)$ \\
\hline $\mathfrak{h}$ & $\left(O_{10}, \Omega_{10}\right)$ \\
\hline $\mathfrak{h} \otimes \tau$ & $\left(O_{8}, \Omega_{8}\right)$ \\
\hline
\end{tabular}

The $W$-equivariant Poincaré polynomial of the coinvariant algebra is equal to

$$
1+\mathfrak{h} \cdot t^{2}+(\mathfrak{h} \otimes \tau) \cdot t^{4}+(\tau+\tau \otimes \sigma) \cdot t^{6}+(\mathfrak{h} \otimes \tau) \cdot t^{8}+\mathfrak{h} \cdot t^{10}+\sigma \cdot t^{12}
$$

therefore

$$
\begin{gathered}
K_{\mathfrak{g}, 1}\left(t^{2}\right)=t^{12}, \quad K_{\mathfrak{g}, \sigma}\left(t^{2}\right)=1, \quad K_{\mathfrak{g}, \tau}=t^{6}=K_{\mathfrak{g}, \tau \otimes \sigma}, \\
K_{\mathfrak{g}, \mathfrak{h}}\left(t^{2}\right)=t^{2}+t^{10}, \quad K_{\mathfrak{g}, \mathfrak{h} \otimes \tau}\left(t^{2}\right)=t^{4}+t^{8} .
\end{gathered}
$$

Thus, Conjecture 8.4 says that

$$
\begin{gathered}
h(\operatorname{triv} ; y)=y^{-12}, \quad h(\sigma ; y)=1, \quad h(\tau ; y)=y^{-6}=h(\tau \otimes \sigma ; y), \\
h(\mathfrak{h} ; y)=y^{-2}+y^{-10}, \quad h(\mathfrak{h} \otimes \tau ; y)=y^{-4}+y^{-8} .
\end{gathered}
$$

The slices to $O_{0}, O_{6}$, and $O_{12}$ have one-dimensional $\mathrm{HP}_{0}$, therefore the conjectural formulas for triv, $\sigma$, and $\tau \otimes \sigma$ follow from Theorem [5.1. The slice to $O_{10}$ is a Kleinian singularity of type $D_{4}$, thus $\operatorname{HP}_{0}\left(X_{O_{10}}\right)$, has the Hilbert series $1+2 t^{4}+t^{8}$. Since $L_{10}$ has rank two, it must occur in weight 4 ; this proves our conjecture for $\tau$ and $\mathfrak{h}$.

Finally, to prove our conjecture for $\mathfrak{h} \otimes \tau$, we need to show that $h\left(\mathrm{HP}_{0}\left(X_{O_{8}}\right) ; t\right)=1+t^{4}$. First note that the dimension of $\mathrm{HP}_{0}\left(X_{O_{8}}\right)$ must be two, as a consequence of [ES10b]. Since $X_{O_{8}}$ is conical and singular, the function $1 \in \mathbf{C}\left[X_{O_{8}}\right]$ has nonzero image in $\operatorname{HP}_{0}\left(X_{O_{8}}\right)$, thus we only need to show that there is a nonzero element of $\operatorname{HP}_{0}\left(X_{O_{8}}\right)$ in degree four. To do this we can use the explicit realization of $X_{O_{8}}$ given in GG02]: it is the intersection of the nilpotent cone with the Slodowy slice $Y:=\Phi(e+\operatorname{ker}(\operatorname{Ad} f))$, with $\Phi: \mathfrak{g} \rightarrow \mathfrak{g}^{*}$ given by the Killing form, with $e \in O_{8}$ and $(e, h, f)$ a corresponding $\mathfrak{s l}_{2}$-triple. Since there is only one nilpotent orbit of dimension 8 , it is easy to see that we can take $e$ to be the generator $e_{\alpha}$ of the root space for the short simple root $\alpha, f=f_{\alpha}$, and $h=h_{\alpha}$. Moreover, as explained in [ES10b], it suffices to compute $\operatorname{HP}_{0}(Y)$ itself, since this is a free module over $\mathbf{C}[\mathfrak{g}]^{\mathfrak{g}}$, with $\mathrm{HP}_{0}\left(X_{O_{8}}\right) \cong \mathrm{HP}_{0}(Y) /\left(\mathbf{C}[\mathfrak{g}]_{+}^{\mathfrak{g}}\right)$, the latter being the augmentation ideal. The latter can be computed explicitly in the first few degrees: under the Kazhdan grading, $\mathbf{C}[Y]$ is a polynomial algebra on generators in degrees $2,2,2,4,5$, and 5 . The first three generators are the $\mathfrak{s l}_{2}$ triple 
mentioned above, and they act trivially on the generator in degree 4 . Thus in degree four, $\mathrm{HP}_{0}(Y)$ has dimension two. However, the generators of $\mathbf{C}[\mathfrak{g}]^{\mathfrak{g}}$ are in degrees four and twelve (these are the fundamental invariants, and the Kazhdan grading restricts on $\mathbf{C}[\mathfrak{g}]^{\mathfrak{g}}$ to the the usual grading placing $\mathfrak{g}^{*}$ in degree two, and the latter is well-known to assign the generators degrees four and twelve). Thus, in degree four, $\mathrm{HP}_{0}\left(X_{O_{8}}\right)$ has dimension one, as desired.

\section{References}

[AL98] Jacques Alev and Thierry Lambre, Comparaison de l'homologie de Hochschild et de l'homologie de Poisson pour une déformation des surfaces de Klein, Algebra and operator theory (Tashkent, 1997), Kluwer Acad. Publ., Dordrecht, 1998, pp. 25-38.

[BBD82] Alexander A. Beilinson, Joseph Bernstein, and Pierre Deligne, Faisceaux pervers, Analysis and topology on singular spaces, I (Luminy, 1981), Astérisque, vol. 100, Soc. Math. France, Paris, 1982, pp. 5-171.

[Bea00] Arnaud Beauville, Symplectic singularities, Invent. Math. 139 (2000), no. 3, 541549.

[BL78] W. M. Beynon and G. Lusztig, Some numerical results on the characters of exceptional Weyl groups, Math. Proc. Cambridge Philos. Soc. 84 (1978), no. 3, 417-426.

[BLPW] Tom Braden, Anthony Licata, Nicholas Proudfoot, and Ben Webster, Quantizations of conical symplectic resolutions II: category $\mathcal{O}$ and symplectic duality, In preparation.

[BM83] Walter Borho and Robert MacPherson, Partial resolutions of nilpotent varieties, Analysis and topology on singular spaces, II, III (Luminy, 1981), Astérisque, vol. 101, Soc. Math. France, Paris, 1983, pp. 23-74.

[BPW] Tom Braden, Nicholas Proudfoot, and Ben Webster, Quantizations of conical symplectic resolutions I: local and global structure, arXiv:1208.3863.

[BS13] Gwyn Bellamy and Travis Schedler, On the (non)existence of symplectic resolutions for imprimitive symplectic reflection groups, arXiv:1309.3558, 2013.

[Car93] Roger W. Carter, Finite groups of Lie type, Wiley Classics Library, John Wiley \& Sons Ltd., Chichester, 1993, Conjugacy classes and complex characters, Reprint of the 1985 original, A Wiley-Interscience Publication.

[Den01] Graham Denham, The combinatorial Laplacian of the Tutte complex, J. Algebra 242 (2001), no. 1, 160-175. 
[EGP $\left.{ }^{+} 12\right]$ Pavel Etingof, Sherry Gong, Aldo Pacchiano, Qingchun Ren, and Travis Schedler, Computational approaches to Poisson traces associated to finite subgroups of $\mathrm{Sp}_{2 n}(\mathbb{C})$, Exp. Math. 21 (2012), no. 2, 141-170.

[ES] Pavel Etingof and Travis Schedler, Coinvariants of Lie algebras of vector fields on algebraic varieties, arXiv:1211.1883.

[ES10a] Poisson traces and D-modules on Poisson varieties, Geom. Funct. Anal. 20 (2010), no. 4, 958-987, With an appendix by Ivan Losev.

[ES10b] _ Traces on finite $\mathcal{W}$-algebras, Transform. Groups 15 (2010), no. 4, 843-850.

[ES13] _ Poisson traces for symmetric powers of symplectic varieties, Int. Math. Res. Not. (2013), doi: 10.1093/imrn/rnt031; arXiv:1109.4712.

[GG02] Wee Liang Gan and Victor Ginzburg, Quantization of Slodowy slices, Int. Math. Res. Not. (2002), no. 5, 243-255.

[Gre75] G.-M. Greuel, Der Gauss-Manin-Zusammenhang isolierter Singularitäten von vollständigen Durchschnitten, Math. Ann. 214 (1975), 235-266.

[Gro70] Alexander Grothendieck, Représentations linéaires et compactification profinie des groupes discrets, Manuscripta Math. 2 (1970), 375-396.

[Har72] Robin Hartshorne, Algebraic de Rham cohomology, Manuscripta Math. 7 (1972), 125-140.

[Har75] R. Hartshorne, On the De Rham cohomology of algebraic varieties, Inst. Hautes Études Sci. Publ. Math. (1975), no. 45, 5-99.

[HS02] Tamás Hausel and Bernd Sturmfels, Toric hyperKähler varieties, Doc. Math. 7 (2002), 495-534 (electronic).

[Kal06] Dmitry Kaledin, Symplectic singularities from the Poisson point of view, J. Reine Angew. Math. 600 (2006), 135-156.

[Kal08] Dmitry Kaledin, Derived equivalences by quantization, Geom. Funct. Anal. 17 (2008), no. 6, 1968-2004.

[Kal09] G Geometry and topology of symplectic resolutions, Algebraic geometrySeattle 2005. Part 2, Proc. Sympos. Pure Math., vol. 80, Amer. Math. Soc., Providence, RI, 2009, pp. 595-628.

[Łoj64] Stanisław Łojasiewicz, Triangulation of semi-analytic sets, Ann. Scuola Norm. Sup. Pisa (3) 18 (1964), 449-474. 
[Lus81] George Lusztig, Green polynomials and singularities of unipotent classes, Adv. in Math. 42 (1981), no. 2, 169-178.

[Lus84] Characters of reductive groups over a finite field, Annals of Mathematics Studies, vol. 107, Princeton University Press, Princeton, NJ, 1984.

[Maf05] Andrea Maffei, Quiver varieties of type A, Comment. Math. Helv. 80 (2005), no. 1, $1-27$.

[Nam] Yoshinori Namikawa, Fundamental groups of symplectic singularities, arXiv:1301.1008.

[Nam08] Yoshinori Namikawa, Flops and Poisson deformations of symplectic varieties, Publ. Res. Inst. Math. Sci. 44 (2008), no. 2, 259-314.

[Pro14] Nicholas Proudfoot, Hypertoric Poisson homology in degree zero, Alg. Geom. 2 (2014), 1-10.

[PW07] Nicholas Proudfoot and Ben Webster, Intersection cohomology of hypertoric varieties, J. Algebraic Geom. 16 (2007), no. 1, 39-63.

[Ste51] R. Steinberg, A geometric approach to the representations of the full linear group over a Galois field, Trans. Amer. Math. Soc. 71 (1951), 274-282.

[Web11] Ben Webster, Singular blocks of parabolic category $\mathcal{O}$ and finite $W$-algebras, J. Pure Appl. Algebra 215 (2011), no. 12, 2797-2804. 